\title{
Need Satisfaction Moderates the Association Between Physical Activity and Affective States in Adults Aged 50+: an Activity-Triggered Ambulatory Assessment
}

\author{
Martina Kanning, $\mathrm{PhD}^{1}$ • Sylvia Hansen, $\mathrm{MSc}^{2}$
}

\begin{abstract}
Background Substantial evidence shows that physical activities of daily living are positively correlated with affective states in middle-aged and older adults. However, people's physical activity decreases when they grow older, and conditions that enhance older individuals' physical activities of daily living are not well understood.

Purpose This study investigated need satisfaction (competence, relatedness, and autonomy) and its moderating effect on the within-subject relation between physical activities of daily living and three dimensions of affective states (valence, energetic arousal, and calmness) based on an ambulatory assessment that used activity-triggered e-diaries.

Method The physical activities of daily living of 68 adults aged $50+($ mean age $=60.1 \pm 7.1)$ were measured objectively for three consecutive days, and need satisfaction and affective states were assessed as a function of the amount of physical activity during the preceding $10 \mathrm{~min}$ before the affect measurement (in activity-triggered e-diaries). Hierarchical multilevel analyses were performed.

Results Need satisfaction was significantly and positively correlated with the three dimensions of affective states. Further, physical activities of daily living were significantly associated with energetic arousal and calmness, but not valence. However, when physical activities of daily living were
\end{abstract}

more autonomously regulated, the association of physical activities of daily living and valence became significant and positive.

Conclusion The findings regarding the significant moderating effects of need satisfaction are crucial for interventions aiming to improve the health-enhancing effects of physical activity in adults aged 50+. Positive feelings owing to physical activities in daily living depend on the extent that psychological needs are satisfied.

Keywords Emotions $\cdot$ Feelings $\cdot$ Self-determination theory Ecological momentary assessment $\cdot$ Subjective well-being · Activities of daily living

In both the USA and several European countries, the baby boomer cohorts will define what we call the elderly. Hence, the proportion of older people will increase, and the old age dependency ratio will be altered to the disadvantage of young people. In this regard, positive affective reactions due to physical activity are relevant for public health initiatives for two reasons: first, positive affective reactions enhance mental health [1]; second, positive feelings and well-being constitute important predictors of the maintenance of physical activity [2]. The maintenance of physical activity is particularly important for adults aged 50+, who can reduce the risk of diseases and premature mortality and secure autonomy by staying active in everyday life and during leisure time [3]. Nevertheless, the total amount of physical activity decreases as people age $[4,5]$.

To enhance positive affective reactions due to physical activity, studies need to investigate the conditions and circumstances that influence the relationship between physical activities and positive affect. This study used psychological need satisfaction based on autonomy, competence, and relatedness 
and analyzed the moderating effect on the abovementioned relationship in daily living situations among people aged $50+$.

Meta-analyses have shown that physical activity is positively associated with different components of psychological wellbeing for adults aged 50+ [6-8]. Moreover, recent ambulatory assessment studies on adults aged 50+ (e.g., [9-13]) have confirmed the positive association of psychological well-being with various physical activities of daily living (e.g., doing household tasks, going for a walk, playing with (grand)children, and shopping for groceries). These physical activities of daily living are most often characterized by reduced intensity and short duration $[14,15]$, and they are particularly relevant as people get older. It has been shown that compared to younger people, with increasing age, the amount of energy expended on leisure time physical activity usually decreases, whereas energy expenditure on household activities increases [16].

According to self-determination theory (SDT), which suggests that people tend to be driven by a need for optimal psychological growth, integrity, and well-being [17], people strive to satisfy three psychological needs: competence, the need to experience mastery and to produce desirable actions; relatedness, the need to feel a connection with or a sense of belonging to the social environment; and autonomy, the need to take actions that are fully integrated into one's own value system. Deci and Ryan [18] conceptualized autonomy as a continuum ranging from intrinsic regulation to extrinsic regulation. In this regard, intrinsic goals are personally valued and autonomous (self-determined), whereas extrinsic goals are controlled and less self-determined. According to SDT, people's subjective well-being is enhanced when their activities are more autonomously regulated and when they experience more feelings of competence and relatedness.

Indeed, many studies have investigated the influence of need satisfaction on well-being and found that well-being is affected by the degree to which behaviors satisfy the three above mentioned psychological needs $[17,19,20]$. A few studies have analyzed within-subject relations between need satisfaction and subjective well-being in daily life. For instance, Reis, Sheldon, Gable, Roscoe, and Ryan [21] investigated daily variations in well-being and showed that such variations arise from the degree to which the three psychological needs are met in situ. In addition, Howell, Chenot, Hill, and Howell [22] demonstrated that momentary psychological need satisfaction is correlated with momentary happiness. These studies analyzed within-subject effects and showed that in addition to a trait component, psychological needs also include a state component. However, both studies used a retrospective diary, which may have influenced participants' ratings of their affective states [23]. To address this limitation, real-time techniques such as an ambulatory assessment method should be used [24].

Since the degree to which behavior (e.g., physical activity) supports psychological needs influences affective states, one could assume that the relation between physical activity and affective states differs according to the extent to which a certain need is satisfied by physical activity. To our knowledge, the moderating effect of need satisfaction with respect to the relation between physical activities of daily living and affective states in daily living situations has been investigated only rarely. For instance, examining autonomy as a moderator between physical activities of daily living and affective states, Kanning, Ebner-Priemer, and Brandt [25] found that the greater extent students engaged in physical activities of daily living and the more this preceding bout of physical activities of daily living was autonomously regulated, the better students in the sample felt. No studies have investigated the moderating role of autonomy in adults aged 50+ or the moderating effects of feelings of competence and relatedness in daily life. However, these are crucial issues for creating effective public health initiatives. If an intervention enhances physical activities of daily living to improve health, people may mainly benefit from its physiological effects such as lower body weight or improved blood pressure. However, if an activity intervention would also consider individuals' need satisfaction, it will strengthen psychological health effects such as improved positive feelings.

The purpose of our study is to analyze the extent to which feelings of autonomy, competence, and relatedness moderate the association between physical activities of daily living and affective states. For this purpose, we first analyzed the main effects of the within-subject relation between need satisfaction (autonomy, competence, and relatedness) and momentary affective states as well as the association between physical activities of daily living and affect. Second, we analyzed the interaction effects of need satisfaction on the relation between physical activities of daily living and affective states. We hypothesized that the association between activity and affect in daily life situations would be greater when the amount of physical activities of daily living was higher and when the preceding bout of physical activities of daily living was (1) more autonomously regulated, (2) characterized by more feelings of competence, or (3) characterized by more feelings of relatedness. Third, we examined cross-level interactions. Because the extent to which individuals engage in physical activity varies by their body weight and gender [26], we analyzed the moderating effects of body mass index (BMI) and gender on the main effects of the within-subject relations. Furthermore, we examined cross-level interactions of the cognitive dimension of well-being (i.e., life satisfaction) and trait components of the three psychological needs.

\section{Method}

\section{Selection of the Study Participants}

This ambulatory assessment study involving activity-triggered e-diaries included 74 adults aged 50+. The authors received 
600 randomly chosen addresses of individuals older than 50 years of age from the registration office of a city in the southern part of Germany. The sample was stratified by age (50 to 70 years old) and sex (with a balanced distribution of men and women). All 600 individuals were contacted, and 74 decided to participate in the study. All of the subjects participated voluntarily, and they received $15 €$ as compensation for their participation. In accordance with the process of institutional ethical approval, all of the participants provided written informed consent: (cf. a further paper of the same dataset: [13]).

Because of technical problems with one of the accelerometers, data for one participant were excluded from the analysis. In addition, data for five people were excluded either because they did not provide any data on the covariates (one person) or because they did not provide any input in the electronic diary (four people). When asked about the missing data, the four individuals cited problems with electronic entry or their inability to hear the acoustic signal. Table 1 presents the characteristics of the study participants.

\section{Ambulatory Assessment with Activity-Triggered e-Diaries}

Given that the amount of physical activity decreases with older age [4, 26], capturing episodes of physical activities of daily living by using a time-based ambulatory assessment (e.g., prompting participants every hour to fill out an electronic diary) is more difficult. To solve this problem, the current study used an ambulatory assessment with activity-triggered e-diaries, as described in another paper [13]. The accelerometer measured the amount of physical activity. We predefined an activity threshold of 220 milli-g (moving average), indicating that the subjects walked at least 6 min during the past $10 \mathrm{~min}$ (walking is, on average, about $350 \mathrm{milli}-\mathrm{g} / \mathrm{min}$ and jogging episodes produce approximately $1000 \mathrm{milli}-\mathrm{g} / \mathrm{min}$ ). We also predefined an inactivity threshold of 10 milli-g

Table 1 Characteristics of study participants

\begin{tabular}{ll}
\hline & $\%$ or mean (SD) \\
\hline Gender (\% men) & $51 \%$ \\
Age & $60.1(7.1)$ \\
Marital status (\% married or de facto) & $65 \%$ \\
Educational attainment & \\
- University degree & $46.4 \%$ \\
Work status & \\
- Currently working $\quad$ Retired & $27.5 \%$ \\
- Homemaker & $53.7 \%$ \\
Physical activity in milli-g/min & $18.7 \%$ \\
$\quad$ (moving average of the 10 & $73.3(35.3) ;$ \\
$\quad$ min before e-diary assessment) & median 70.9 \\
\hline
\end{tabular}

(moving average), which would be equivalent to the acceleration of sitting. When these thresholds were surpassed, the accelerometer made an acoustic sound, and the subjects were asked to fill out the e-diary. We did not program a reminder, so data were missing if the participant did not answer. We kept the data between 8 a.m. and 9 p.m. and programmed a minimum time interval of $40 \mathrm{~min}$ and a maximum time interval of $100 \mathrm{~min}$ between the diary assessments (for more details, see [27]).

\section{Procedure}

The study was conducted over the course of three consecutive days, beginning on Thursday and ending on Saturday in order to include both week and weekend days. We investigated only 3 days to minimize the burden of the assessment. Using an activity-triggered e-diary gives us the possibility of capturing more physically active episodes than using a normal ambulatory assessment. Research assistants visited the participants in their homes on Thursday. The assistants explained the details of the study design and asked the participants to sign an informed consent form. The research assistants explained and demonstrated how to use the accelerometer and the smart phone, and the accelerometer was placed on a belt on the individual's hip. Finally, the subjects completed a questionnaire that included items on their demographics and quality of life.

\section{Measures}

\section{Physical Activities of Daily Living}

We used the varioport-e (Becker, Meditech, Karlsruhe, Germany) as an accelerometer, which enables real-time analyses. The varioport-e measures and stores acceleration on three axes with a frequency of $64 \mathrm{~Hz}$. The raw acceleration data (milli-g) were separated offline into AC and DC components by using a FIR digital filter, which has a cutoff frequency of $0.5 \mathrm{~Hz}$. The raw signals, DC values, and rectified AC values were then averaged across data points for each minute during the 3-day measurement period. All of the offline analyses and artifact checks were performed by using an interactive software package (Freiburg Monitoring System) according to a published procedure [28]. To analyze the association between physical activities of daily living and affective states, the physical activities of daily living values during the $10 \mathrm{~min}$ preceding each entry into the e-diary were averaged.

\section{Affective States}

Affective states represent the affective component. Together with the cognitive component (life satisfaction), they constitute the construct of subjective well-being, which is mainly used to operationalize mental health. Affective states are a 
multidimensional construct that contains dimensions for at least valence (pleasure-displeasure) and arousal (tired-awake) [29, 30]. However, based on findings from structural equation analyses, Schimmack and Reisenzein [31] demonstrated that arousal should be differentiated into the following two dimensions: power (full of energy-without energy) and excitation (calmagitated). Thus, together with valence (pleasure-displeasure), affective states involve three dimensions of affect. In this study, we used the Short Mood Scale, based on the Multidimensional Mood Questionnaire, for which evidence of validity and reliability has been demonstrated (MDMQ; [32]). The Short Mood Scale distinguishes between three dimensions of affective states and is the only instrument that has been explicitly developed and evaluated for use in ambulatory assessment [33]. The scale contains six items that assess the intensity of the three dimensions of affective states, which are ordered as semantic differentials: valence (unwell vs. well, discontent vs. content), calmness (relaxed vs. tense, calm vs. agitated), and energetic arousal (tired vs. awake, without energy vs. full of energy). In this study, the subjects responded to the prompt "At this moment, I feel..." by moving a slider from the left end (e.g., unwell) to the right end (e.g., well) of a bipolar scale. Scores for each subscale were obtained by averaging the item scores, which resulted in a range from zero to five. Note that the sevenpoint Likert scale of the original version was changed to a sixpoint scale, which did not contain a mean value, to force the subjects to decide on a direction for each dimension (e.g., unwell vs. well). Wilhelm and Schoebi [33] investigated the homogeneity of the scale items at both the between-subject and the within-subject levels. The reliability coefficient for the between-subject level was 0.92 for valence and 0.90 for energetic arousal and calmness, whereas the reliability coefficient for the within-subject level was 0.70 for valence and calmness and 0.77 for energetic arousal. Both reliability coefficients thus indicate satisfactory internal consistency.

\section{Autonomy}

We assessed the psychological need for momentary autonomy with the Relative Autonomy Index (RAI). The RAI was proposed by Sheldon and Elliot [20], and the corresponding scale has been shown to have a high level of internal consistency (Cronbach's $\alpha=0.80$ ). The participants were asked to rate items concerning possible regulation modes of their ongoing activities. After the prompt "I was engaged in this activity over the last 10 min because...," the participants responded to the four items "I just felt like doing it" (intrinsic regulation), "I believe these activities were meaningful and important" (identified regulation), "I didn't want to be blamed by someone" (introjected regulation), and "I wanted to be recognized by others for doing that" (external regulation) on six-point scales ranging from zero (not at all for this reason) to five (solely for this reason). In line with recent recommendations for scoring this scale [21], the RAI was calculated by adding intrinsic and identified regulation scores and subtracting the added introjected and external regulation scores.

\section{Competence}

The psychological need for momentary competence was measured with the same single item that Reis et al. [21] used: "I felt capable and competent during my activities of the last 10 min." The participants responded to this item by using a six-point scale ranging from zero (completely disagree) to five (completely agree).

\section{Relatedness}

To assess the psychological need for relatedness, we concentrated on the issue of being alone versus being together with someone. For this measure, we used a dichotomous scale. The participants thus responded to the prompt "During the last 10 min, I was mainly ..."alone" or "together with others."

\section{Covariates}

The moderating effects of the following four variables were assessed: gender and BMI (using self-reported body weight and height). Third, the impact of general satisfaction with life, which is the cognitive component of subjective well-being, was assessed using the Satisfaction with Life Scale (SWLS) developed by Diener, Emmons, Larsen, and Griffin [34]. Pavot and Diener [35] confirmed moderate to high internal consistencies (Cronbach's alpha 0.79 to 0.89 ) and showed good convergent validity with related scales and discriminant validity from emotional well-being measures. The scale contains five items (e.g., "If I could live my life over, I would change almost nothing"), and the participants answered these items by using a five-point response scale that ranged from completely disagree [1] to completely agree [5]. The ratings for the five items were averaged to compose the satisfaction with one's life measure. Fourth, the basic psychological need satisfaction scale was used to assess general need satisfaction of autonomy (seven items, Cronbach's alpha $=0.69$; e.g., I feel like I am free to decide for myself how to live my life), competence (six items, Cronbach's alpha $=0.71$; e.g., People I know tell me I am good at what I do), and relatedness (eight items, Cronbach's alpha $=0.69$; I consider the people I regularly interact with to be my friends) with 21 items [36]. The authors reported high internal consistency associated with the total need satisfaction score. The participants rated on a sevenpoint rating scale the extent to which they are generally satisfied in their life with respect to psychological needs. We treated need satisfaction as a multidimensional construct and analyzed the three psychological needs separately. 


\section{Data Analyses}

To analyze the within-subject relations between physical activities of daily living, affective states, and need satisfaction as well as the impact of the covariates (cross-level interactions), we must consider the hierarchical structure of the data. The multiple measures of momentary affective states, physical activities of daily living, and psychological need satisfaction define the lower level of the hierarchy, and these components were nested within the subjects who define the higher level of the hierarchy. Multilevel analyses were applied by using the HLM 6.0 statistical program [37], and restricted maximum likelihood estimations were used, with an alpha level of $p<0.05$.

A step-up approach was used [38]. First, for each affect subscale (valence, energetic arousal, and calmness) in the absence of predictor variables, a model was tested to separate the variance into within- and between-subject sources. For this purpose, an estimate of the intraclass correlations was used. In the second step, level 1 predictor variables (physical activity of daily living, psychological need satisfaction, and their interactions) were consecutively entered into each model of the affect subscale separately. In the final step, we analyzed whether valence, energetic arousal, calmness, the last $10 \mathrm{~min}$ of physical activities of daily living (PA) before each e-diary entry, or satisfaction with respect to autonomy (Aut), competence (Comp), and relatedness (Rel) significantly varied as a function of sex (SEX), BMI, basic need satisfaction (BPNS), and general satisfaction with life (SWLS). We did not assess a cross-level interaction for the three interaction terms of level 1 (Aut $\times$ PA, Comp $\times$ PA, and Rel $\times$ PA). The equations are as follows:

$$
\begin{array}{ll}
\text { Level 1: } & Y_{t i}=b_{0 i}+b_{1 i}(\mathrm{PA})_{t i}+b_{2 i}(\mathrm{Aut})_{t i}+b_{3 i}(\mathrm{Comp})_{t i}+ \\
& b_{4 i}(\operatorname{Rel})_{t i}+b_{5 i}(\text { Aut x PA })_{t i}+b_{6 i}(\text { Comp x PA })_{t i}+ \\
& b_{7 i}(\text { Rel x PA })_{t i}+r_{t i}
\end{array}
$$

Level $2($ Intercept $): \quad b_{0 i}=\gamma_{00}(\mathrm{SEX})+\gamma_{01}(\mathrm{BMI})$

$$
+\gamma_{02}(\mathrm{SWLS})+\mu_{0 i}
$$

Level $2(\mathrm{PA}): b_{1 i}=\gamma_{10}(\mathrm{SEX})+\gamma_{11}(\mathrm{BMI})$

$$
+\gamma_{12}(\mathrm{SWLS})+\mu_{1 i}
$$

Level 2 (Aut) $: b_{2 i}=\gamma_{20}($ SEX $)+\gamma_{21}($ SWLS $)$

$$
+\gamma_{22}(\text { BPNS_Aut })+\mu_{2 i}
$$

Level $2($ Comp $): b_{3 i}=\gamma_{30}($ SEX $)+\gamma_{31}($ SWLS $)$

$$
+\gamma_{32}(\text { BPNS_Comp })+\mu_{3 i}
$$

Level $2($ Rel $): b_{4 i}=\gamma_{40}($ SEX $)+\gamma_{41}($ SWLS $)$

$$
+\gamma_{42}(\text { BPNS_Rel })+\mu_{4 i}
$$

Level 1 calculates the within-subject effects. Equation 1 represents a subject's response (subscript ${ }_{i}$ ) for one of the three subscales $\left(\mathrm{Y}_{t i}\right)$ for any given diary entry (subscript $\left.{ }_{t}\right)$. $Y_{t i}$ is defined as the average intercept of one subscale across all of the subjects $\left(b_{0 i}\right)$ and the seven level 1 predictors. These predictors are mean centered by group, where the group refers to a person. With this calculation, we can disaggregate the between- and within-subject effects [39]. The intercepts and slopes vary randomly. The random effect for the level 1 model is given by $r_{t i}$, which is assumed to have a normal distribution, a mean of " 0 " and a variance of $\sigma^{2}$.

Level 2 calculates the between-subject effects. This level includes the fixed effects, three covariates, and the random effects. The random effects are assumed to be multivariate and normally distributed, with both having expected values of 0 .

To clarify the magnitude of the effects, the seven level 1 predictors (PA, Aut, Comp, Rel, Aut $\times$ PA, Comp $\times$ PA, and Rel $\times$ PA) on the three subscales of momentary affective states were standardized (see Eqs. 7 and 8). Standard deviations were obtained from the mean of the sample for each level 1 predictor and from the averaged within-subject mean of the affect subscale. The standardized effect was calculated as follows:

Standardized effect : $b_{1 \mathrm{i}} * \mathrm{SD}(\mathrm{PA}$, Aut, Rel, Aut $\times$ PA, Comp $\times$ PA, Rel $\times \mathrm{PA}) / \mathrm{SD}($ correspondent affect subscale)

For the dichotomous variable competence, the standardized effect was calculated as follows:

Standardized effect $b_{1 \mathrm{i}}$ (Comp)/SD (correspondent affect subscale)

\section{Results}

\section{Descriptive Statistics}

The 68 participants provided 1311 data points, with an average of 19.3 e-diary entries per subject. All assessments were completed within 10 min of the prompt. All in all, fewer than $10 \%$ of the total possible numbers of assessments were missing. The intraclass coefficients were $\rho_{\mathrm{I}}=0.30, \rho_{\mathrm{I}}=0.21$, and $\rho_{\mathrm{I}}=0.26$ for valence, energetic arousal, and calmness, respectively, indicating that $70 \%, 79 \%$, and $74 \%$ of the variance for the respective affect subscales were caused by intraindividual variation (e.g., owing to situational effects). The moving average of the 10-min physical activity before e-diary assessments ranged from 0 to 1308 milli-g/min, with $25 \%$ of the activity values being lower than 13 milli-g/min, $50 \%$ being lower than 
$71 \mathrm{milli}-\mathrm{g} / \mathrm{min}$ (median), and $75 \%$ being lower than 148 milli$\mathrm{g} / \mathrm{min}$. In general, participants in the sample were inactive or active with a low intensity, which is comparable with the activity of such an age group [40]. Descriptive data for the variables of the analysis are reported in Table 2. The distribution of these variables allowed for a multilevel analysis. The fixed and random effects for each dimension of momentary affective states are displayed in Table 3. In the following sections, we describe the main and interaction effects and the cross-level interactions of the covariates for each dimension of affect separately.

\section{Affect Subscale of Valence}

Against our expectations, valence was not significantly predicted by the last $10 \mathrm{~min}$ of physical activities of daily living before each diary entry but was significantly predicted by all three psychological needs, which we expected. The more the subjects experienced feelings of autonomy, competence, or relatedness, the more they felt well and content. Overall, $86 \%, 80 \%$, and $64 \%$ of the slopes for autonomy, competence, and relatedness were positive, respectively. According to the standardized effects, if autonomy or competence increased by $1 \mathrm{SD}$, valence increased by $0.01 \mathrm{SD}$ or $0.15 \mathrm{SD}$, respectively. Furthermore, in situations, in which a person had indicated that he or she was together with other people (cf. relatedness "being together"), valence was 0.15 SD higher compared to situations, in which this person indicated that he or she was alone (cf. relatedness "being alone"). In addition to the main effects at level 1 , the interaction of autonomy with physical activity in daily living was significant, as hypothesized. That means, the extent to which the activity of the last 10 min was autonomously regulated moderated the relationship between physical activities of daily living and valence (standardized effect $=0.01$ ).

Table 2 Descriptive data of the psychological constructs and physical activity (PA)
Neither the level of valence nor the main effects at level 1 differed between men and women. There was nevertheless a significant cross-level interaction for SWLS for the intercept and for the within-subject effect of competence on valence. When the sample was more satisfied with their lives, they were more likely to feel well and content across all daily living situations. Furthermore, the association between competence and valence varied by life satisfaction.

To illustrate the significant within-subject interactions, we used R [41]. We plotted simple slopes to illustrate the impact of autonomy. One line represents situations in which participants rated their autonomous regulation one standard deviation above the mean of autonomy, one line represents the mean, and the third line represents one standard deviation below the mean of autonomy. Figure 1 illustrates the significant interaction effect: The more the subjects engaged in physical activities of daily living and the more this bout of activity was autonomously regulated, the more the subjects felt well and content. The interaction effect seems to be greater, the higher the volume of everyday life activity is. However, moderate to vigorous activities are rare in everyday life; thus, evidence for this finding was based on very few data.

\section{Affect Subscale of Energetic Arousal}

Energetic arousal was significantly and positively associated with the last $10 \mathrm{~min}$ of physical activities of daily living and with all three psychological needs, as anticipated. The more the subjects were physically active, the more they experienced feelings of autonomy and competence. Moreover, when they were together with others during the preceding bout of physical activities of daily living, they were more likely to report feeling awake and full of energy. Most of the slopes were positive for physical activities of daily living (80\%), autonomy $(66 \%)$, and competence $(81 \%)$. According to the

\begin{tabular}{lllll}
\hline Variable & Mean (SD) & Min & Max & Intraclass coefficient \\
\hline Level 1 (assessed in situations) & & & & \\
$\quad$ Energetic arousal & $3.57(1.16)$ & 0 & 5 & 0.21 \\
$\quad$ Valence & $4.02(0.95)$ & 0 & 5 & 0.30 \\
Calmness & $3.64(1.08)$ & 0 & 5 & 0.26 \\
PA & $105.76(138.09)$ & 0.00 & 1307.00 & \\
Autonomy & $4.47(3.97)$ & -10 & 10 & \\
Competence & $3.96(1.34)$ & 0 & 5 & \\
Relatedness (dichotomous) & $0.42(0.49)$ & 0 & 1 & \\
Level 2 (assessed in persons) & & & & \\
Satisfaction with life (SWLS) & $3.89(0.61)$ & 2.20 & 5.00 & \\
BPNS_Autonomy & $3.88(0.50)$ & 2.50 & 4.80 & \\
BPNS_Competence & $4.03(0.59)$ & 2.70 & 5.00 & \\
BPNS_Relatedness & $3.98(0.46)$ & 2.90 & 4.90 & \\
\hline
\end{tabular}


Table 3 Fixed and random effects and variance components for valence (model 1), energetic arousal (model 2), and calmness (model 3) on physical activity (PA), the three psychological needs (autonomy, competence, and relatedness), and their interactions

\begin{tabular}{|c|c|c|c|c|c|c|c|c|c|c|c|c|}
\hline \multirow{2}{*}{$\begin{array}{l}\text { Outcome } \\
\text { Predictor }\end{array}$} & \multicolumn{5}{|l|}{ Fixed } & \multicolumn{7}{|l|}{ Random } \\
\hline & Coefficient & $\mathrm{SE}$ & $t$ value & $d f$ & $p$ value & SD & c2 & $d f$ & $p$ value & \multicolumn{2}{|c|}{$\begin{array}{l}95 \% \text { Predictive } \\
\text { interval }^{\mathrm{a}}\end{array}$} & $\begin{array}{l}\text { Slopes }>0^{\mathrm{b}} \\
(\%)\end{array}$ \\
\hline \multicolumn{13}{|l|}{ Model 1: valence } \\
\hline Intercept & 4.01 & 0.06 & 61.76 & 63 & $<0.001$ & 0.51 & 195.68 & 25 & $<0.001$ & & & \\
\hline Sex & -0.01 & 0.14 & -0.05 & 63 & 0.96 & & & & & & & \\
\hline BMI & 0.03 & 0.02 & 1.42 & 63 & 0.16 & & & & & & & \\
\hline SWLS & 0.33 & 0.11 & 3.30 & 63 & 0.01 & & & & & & & \\
\hline aPA & 0.000076 & 0.000262 & 0.29 & 63 & 0.77 & 0.00113 & 48.14 & 25 & 0.01 & -0.0021 & 0.0023 & 53 \\
\hline $\mathrm{aPA} \times \operatorname{sex}$ & 0.000740 & 0.000543 & -1.36 & 63 & 0.18 & & & & & & & \\
\hline $\mathrm{aPA} \times \mathrm{BMI}$ & -0.000037 & 0.000086 & -0.42 & 63 & 0.67 & & & & & & & \\
\hline aPA $\times$ SWLS & -0.000021 & 0.000371 & -0.06 & 63 & 0.96 & & & & & & & \\
\hline Autonomy & 0.06 & 0.01 & 6.18 & 63 & $<0.001$ & 0.05 & 53.72 & 25 & 0.01 & -0.05 & 0.16 & 86 \\
\hline Aut $\times \operatorname{sex}$ & -0.01 & 0.02 & -0.36 & 63 & 0.72 & & & & & & & \\
\hline Aut $\times$ SWLS & -0.02 & 0.02 & -0.95 & 63 & 0.35 & & & & & & & \\
\hline Aut $\times$ BPNS_Aut & 0.03 & 0.01 & 1.94 & 63 & 0.06 & & & & & & & \\
\hline Competence & 0.17 & 0.04 & 4.79 & 63 & $<0.001$ & 0.19 & 115.67 & 25 & $<0.001$ & -0.22 & 0.56 & 80 \\
\hline Com $\times$ sex & -0.06 & 0.07 & -0.91 & 63 & 0.37 & & & & & & & \\
\hline Com $\times$ SWLS & -0.12 & 0.06 & -2.09 & 63 & 0.04 & & & & & & & \\
\hline Com $\times$ BPNS_Com & 0.04 & 0.06 & 0.58 & 63 & 0.56 & & & & & & & \\
\hline Relatedness & 0.14 & 0.07 & 2.06 & 63 & 0.04 & 0.39 & 68.78 & 25 & $<0.001$ & -0.62 & 0.89 & 64 \\
\hline Rel $\times \operatorname{sex}$ & -0.12 & 0.13 & -0.89 & 63 & 0.38 & & & & & & & \\
\hline Rel $\times$ SWLS & -0.13 & 0.11 & -1.22 & 63 & 0.23 & & & & & & & \\
\hline Rel $\times$ BPNS_Rel & 0.11 & 0.14 & 0.81 & 63 & 0.42 & & & & & & & \\
\hline Interaction $\mathrm{aPA} \times$ Aut & 0.000262 & 0.000097 & 2.71 & 66 & 0.01 & 0.00043 & 76.49 & 28 & $<0.001$ & -0.0006 & 0.0011 & 73 \\
\hline Interaction aPA $\times \mathrm{Com}$ & -0.000157 & 0.000291 & -0.54 & 66 & 0.59 & 0.00108 & 43.78 & 28 & 0.03 & -0.0023 & 0.0020 & 44 \\
\hline Interaction aPA $\times$ Rel & -0.000155 & 0.000407 & -0.38 & 66 & 0.71 & 0.00070 & 43.86 & 28 & 0.03 & -0.0015 & 0.0012 & 41 \\
\hline \multicolumn{13}{|c|}{ Model 2: energetic arousal } \\
\hline Intercept & 3.59 & 0.07 & 51.58 & 63 & $<0.001$ & 0.52 & 411.28 & 58 & $<0.001$ & & & \\
\hline Sex & -0.11 & 0.16 & -0.71 & 63 & 0.48 & & & & & & & \\
\hline BMI & 0.02 & 0.03 & 0.83 & 63 & 0.41 & & & & & & & \\
\hline SWLS & 0.31 & 0.11 & 2.71 & 63 & 0.01 & & & & & & & \\
\hline aPA & 0.001896 & 0.000450 & 4.22 & 63 & $<0.001$ & 0.00228 & 104.06 & 58 & $<0.001$ & -0.0026 & 0.0064 & 80 \\
\hline $\mathrm{aPA} \times \operatorname{sex}$ & 0.001268 & 0.001022 & 1.24 & 63 & 0.23 & & & & & & & \\
\hline $\mathrm{aPA} \times \mathrm{BMI}$ & 0.000221 & 0.000165 & 1.34 & 63 & 0.18 & & & & & & & \\
\hline aPA $\times$ SWLS & -0.000184 & 0.000712 & -026 & 63 & 0.78 & & & & & & & \\
\hline Autonomy & 0.03 & 0.01 & 2.06 & 63 & 0.04 & 0.06 & 105.93 & 58 & $<0.001$ & -0.09 & 0.14 & 66 \\
\hline Aut $\times \operatorname{sex}$ & 0.01 & 0.02 & 0.09 & 63 & 0.93 & & & & & & & \\
\hline Aut $\times$ SWLS & 0.03 & 0.02 & 1.42 & 63 & 0.16 & & & & & & & \\
\hline Aut $\times$ BPNS_Aut & -0.03 & 0.02 & -1.31 & 63 & 0.19 & & & & & & & \\
\hline Competence & 0.21 & 0.04 & 4.54 & 63 & $<0.001$ & 0.23 & 131.65 & 58 & $<0.001$ & -0.25 & 0.65 & 81 \\
\hline Com $\times$ sex & -0.19 & 0.09 & -2.25 & 63 & 0.03 & & & & & & & \\
\hline Com $\times$ SWLS & -0.09 & 0.07 & -1.34 & 63 & 0.19 & & & & & & & \\
\hline Com $\times$ BPNS_Com & 0.19 & 0.09 & 2.26 & 63 & 0.03 & & & & & & & \\
\hline Relatedness & 0.11 & 0.06 & 1.93 & 1277 & 0.05 & & & & & & & \\
\hline Interaction aPA $\times$ Aut & 0.000401 & 0.000099 & 4.04 & 1277 & $<0.001$ & & & & & & & \\
\hline Interaction aPA $\times \mathrm{Com}$ & -0.000963 & 0.000290 & -3.33 & 1277 & 0.01 & & & & & & & \\
\hline Interaction aPA $\times$ Rel & -0.000676 & 0.000557 & -1.21 & 1277 & 0.23 & & & & & & & \\
\hline
\end{tabular}


Table 3 (continued)

\begin{tabular}{|c|c|c|c|c|c|c|c|c|c|c|c|c|}
\hline \multirow{2}{*}{$\begin{array}{l}\text { Outcome } \\
\text { Predictor }\end{array}$} & \multicolumn{5}{|l|}{ Fixed } & \multicolumn{7}{|l|}{ Random } \\
\hline & Coefficient & $\mathrm{SE}$ & $t$ value & $d f$ & $p$ value & SD & $\mathrm{c} 2$ & $d f$ & $p$ value & $\begin{array}{l}95 \% \text { Pre } \\
\text { interval }^{\mathrm{a}}\end{array}$ & lictive & $\begin{array}{l}\text { Slopes }>0^{\mathrm{b}} \\
(\%)\end{array}$ \\
\hline \multicolumn{13}{|l|}{ Model 3: calmness } \\
\hline Intercept & 3.65 & 0.07 & 51.59 & 63 & $<0.001$ & 0.54 & 573.38 & 55 & $<0.001$ & & & \\
\hline Sex & -0.04 & 0.16 & -0.27 & 63 & 0.79 & & & & & & & \\
\hline BMI & 0.04 & 0.03 & 1.49 & 63 & 0.14 & & & & & & & \\
\hline SWLS & 0.37 & 0.12 & 3.17 & 63 & 0.01 & & & & & & & \\
\hline $\mathrm{aPA}$ & -0.001923 & 0.000471 & -4.09 & 63 & $<0.001$ & 0.00275 & 153.28 & 55 & $<0.001$ & -0.0073 & 0.0035 & 64 \\
\hline $\mathrm{aPA} \times \operatorname{sex}$ & -0.001021 & 0.001061 & -0.96 & 63 & 0.34 & & & & & & & \\
\hline $\mathrm{aPA} \times \mathrm{BMI}$ & -0.000022 & 0.000174 & -0.13 & 63 & 0.89 & & & & & & & \\
\hline $\mathrm{aPA} \times \mathrm{SWLS}$ & 0.000131 & 0.000763 & 0.17 & 63 & 0.86 & & & & & & & \\
\hline Autonomy & 0.07 & 0.01 & 6.67 & 63 & $<0.001$ & 0.06 & 97.79 & 55 & 0.01 & -0.04 & 0.18 & 89 \\
\hline Aut $\times \operatorname{sex}$ & -0.01 & 0.02 & -0.05 & 63 & 0.96 & & & & & & & \\
\hline Aut $\times$ SWLS & 0.01 & 0.02 & 0.44 & 63 & 0.66 & & & & & & & \\
\hline Aut $\times$ BPNS_Aut & 0.01 & 0.02 & 0.17 & 63 & 0.87 & & & & & & & \\
\hline Competence & 0.09 & 0.04 & 2.53 & 63 & 0.01 & 0.19 & 100.52 & 55 & $<0.001$ & -0.27 & 0.46 & 69 \\
\hline Com $\times \operatorname{sex}$ & -0.01 & 0.07 & -0.11 & 63 & 0.91 & & & & & & & \\
\hline Com $\times$ SWLS & -0.09 & 0.06 & -1.46 & 63 & 0.15 & & & & & & & \\
\hline Com $\times$ BPNS_Com & 0.05 & 0.08 & 0.62 & 63 & 0.54 & & & & & & & \\
\hline Relatedness & 0.15 & 0.07 & 2.07 & 63 & 0.04 & 0.38 & 74.24 & 55 & 0.04 & -0.60 & 0.91 & 65 \\
\hline $\operatorname{Rel} \times \operatorname{sex}$ & -0.14 & 0.15 & -0.92 & 63 & 0.36 & & & & & & & \\
\hline Rel $\times$ SWLS & 0.02 & 0.12 & 0.17 & 63 & 0.86 & & & & & & & \\
\hline Rel $\times$ BPNS_Rel & -0.11 & 0.16 & -0.66 & 63 & 0.51 & & & & & & & \\
\hline Interaction aPA $\times$ Aut & 0.000091 & 0.000088 & 1.03 & 1274 & 0.30 & & & & & & & \\
\hline Interaction aPA $\times \mathrm{Com}$ & -0.000112 & 0.000247 & -0.45 & 1274 & 0.65 & & & & & & & \\
\hline Interaction aPA $\times$ Rel & 0.001082 & 0.000500 & 2.16 & 1274 & 0.03 & & & & & & & \\
\hline
\end{tabular}

$P A$ physical activity, Aut autonomy, Com competence, Rel relatedness, BMI body mass index, SWLS Satisfaction with Life Scale, BPNS Basic Psychological Needs Scale

${ }^{\text {a }}$ Based on the assumption of normally distributed regression coefficients, the $95 \%$ predictive interval indicates the range of values between which $95 \%$ of the regression coefficients are estimated to lie (Hox 2010). The intervals were calculated based on a model without level 2 predictors

${ }^{\mathrm{b}}$ Based on the assumption of normally distributed regression coefficients, this value indicates the percentages of regression coefficients that are positive (Hox 2010). The percentages were calculated based on a model without level 2 predictors

standardized effects, if physical activities of daily living increased by $1 \mathrm{SD}$, energetic arousal increased by $0.06 \mathrm{SD}$. Further, if autonomy or competence increased by $1 \mathrm{SD}$, energetic arousal increased by $0.01 \mathrm{SD}$ or $0.18 \mathrm{SD}$, respectively. Furthermore, in situations being together (relatedness), energetic arousal is $0.09 \mathrm{SD}$ higher compared to situations of being alone. In addition to the main effects, the interactions between autonomy and physical activities of daily living (standardized effect $=0.02$ ) and between competence and physical activities of daily living (standardized effect $=-0.001$ ) were significant.

SWLS was associated with the level of energetic arousal. Specifically, the more the subjects were satisfied with their lives, the more they felt full of energy and awake across all daily living situations. Gender was not associated with the general level of energetic arousal, but it showed a significant cross-level interaction with the within-subject effect of competence. In addition, the basic psychological need satisfaction for competence interacted with this within-subject effect.

To illustrate both of the significant within-subject interactions, we used simple slopes for both autonomy and competence and plotted two separate graphs similar to the graph for valence. The graph of Fig. 2 shows that the more the subjects engaged in daily physical activities and the more this bout of physical activity was autonomously regulated, the more the subjects felt full of energy and awake. The effect seems to grow with increasing volumes of activity. But, as already mentioned for the interaction effect on valence, the analysis was based on very few activity data.

Figure 3 illustrates that the more the subjects engaged in physical activities of daily living and the more the subjects 


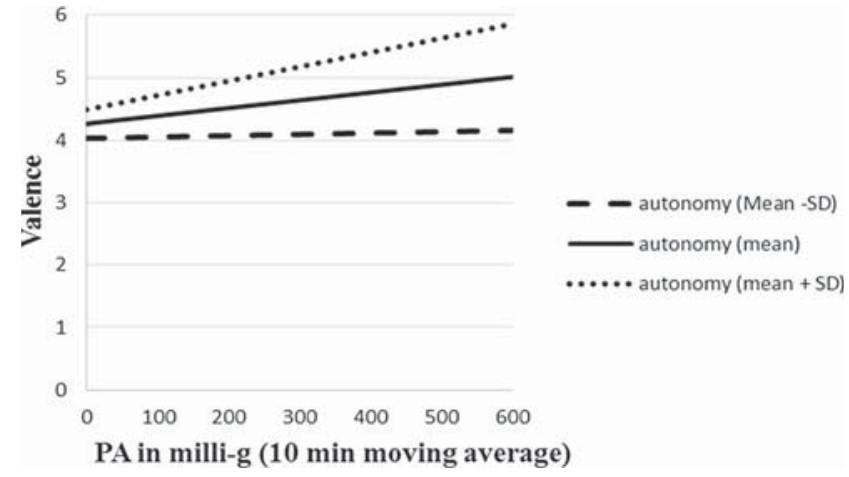

Fig. 1 Within-subject interaction of autonomy and PA on valence. To illustrate how autonomy affected the association between the last $10 \mathrm{~min}$ of PA and valence, we calculated simple slopes

experienced feelings of competence, the more likely the subjects were to feel tired.

\section{Affect Subscale of Calmness}

Calmness was significantly and negatively predicted by the last 10 min of physical activities of daily living (standardized effect $=-0.06$ ) and positively predicted by the three psychological needs (standardized effect for autonomy $=0.01$; standardized effect for competence $=0.08$; standardized effect for being together $=0.14$ ). The subjects were more likely to feel agitated and tense when they were more physically active during the $10 \mathrm{~min}$ before each e-diary entry, as indicated by the fact that $76 \%$ of the slopes for physical activities of daily living were negative. Moreover, the subjects were more likely to feel relaxed and calm when they reported greater satisfaction of their psychological needs, as $89 \%, 69 \%$, and $65 \%$ of the slopes for autonomy, competence, and relatedness, respectively, were positive. In addition to the main effects of the withinsubject relation, the interaction between relatedness and physical activities of daily living was significant (standardized effect $=0.008$ ). SWLS was also significantly and positively

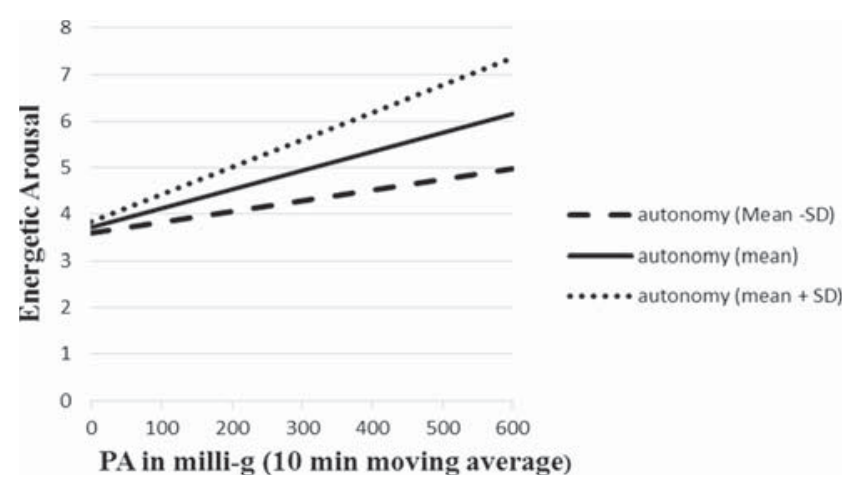

Fig. 2 Within-subject interaction of autonomy and PA on energetic arousal. To illustrate how autonomy affected the association between the last $10 \mathrm{~min}$ of PA and energetic arousal, we calculated simple slopes

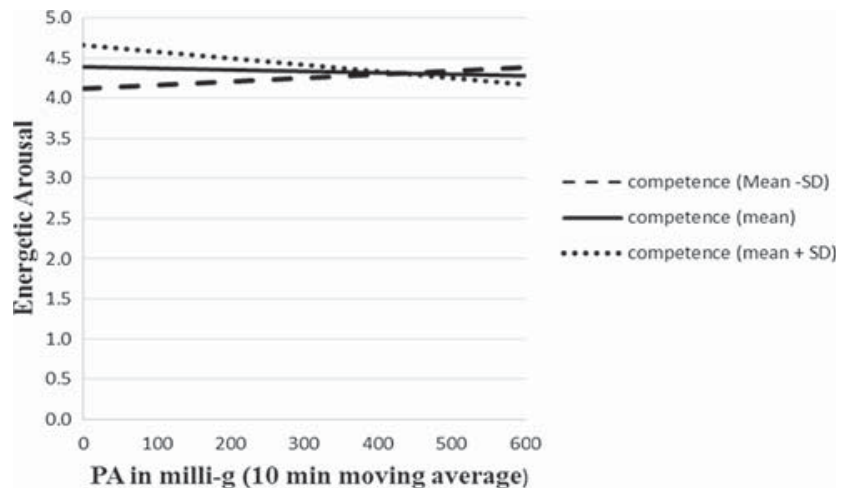

Fig. 3 Within-subject interaction of competence and PA on energetic arousal. To illustrate how competence affected the association between the last 10 min of PA and energetic arousal, we calculated simple slopes

related to the level of calmness. However, gender did not exhibit any significant relationships.

Again, we illustrated the within-subject interaction and plotted two regression lines for situations characterized by "being alone" versus situations characterized by "being together with others." Figure 4 illustrates that the participants felt more agitated and tense when they had been more physically active and when they felt more alone.

\section{Discussion}

This study expanded existing research by analyzing the moderating effect of three psychological needs on the withinsubject relation between physical activities of daily living and affective states in daily living situations among adults aged 50+. Considering the significant interaction effects, the positive association between physical activities of daily living and affective states seems to be moderated by the degree to which feelings of autonomy, competence, and belonging are satisfied.

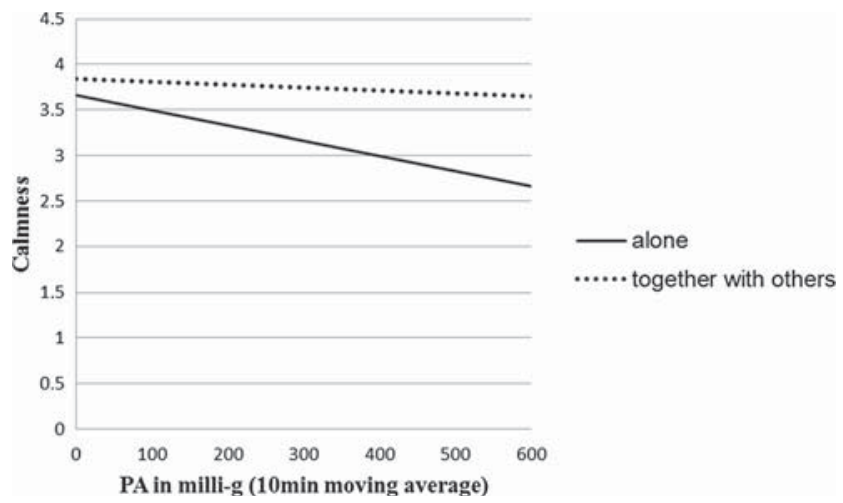

Fig. 4 Within-subject interaction of relatedness and PA on calmness. To illustrate how relatedness affected the association between the last $10 \mathrm{~min}$ of PA and calmness, we calculated separate regression lines for situations in which participants indicated to be alone or to be together with others during the last $10 \mathrm{~min}$ 
In line with the assumptions of SDT and with findings from previous studies $[17,19,20]$, our results show that psychological need satisfaction is positively associated with all three dimensions of affective states. Our findings also indicate that need satisfaction is relevant for affective states in daily living situations (cf. [25]). Reis et al. [21] obtained results supporting such a conclusion by examining the relation between daily variations in well-being and daily psychological need satisfaction for a sample of students who had to retrospectively rate their well-being and need satisfaction before going to bed. In addition, Howell et al. [22] conducted a time-based ambulatory assessment to investigate the association between momentary happiness (in terms of enjoyment and stress) and momentary psychological need satisfaction among 144 undergraduate students. They found a positive association between momentary happiness and both autonomy and relatedness but, in contrast to our findings, a negative association between momentary happiness and competence. To explain this finding, the authors noted that "competence-producing behaviors appear to come at a momentary emotional cost" ([22], p. 12). Thus, compared with students, who fill their days mainly with competence-producing behaviors (e.g., learning activities), adults aged 50+ may not be as involved in such behaviors. This difference may explain the different moderating effects between younger and older people.

The second main effect concerning the association between physical activities of daily living and affective states in daily living situations is generally consistent with previous ambulatory assessment studies on adults aged 50+. Such individuals feel not only more energetic (e.g., [10-12, 42]) but also more agitated (e.g., [43] in a sample of students) after engaging in physical activity in daily living situations. In contrast to these previous studies, we did not find a significant association for valence. Adults aged 50+ were not more likely to feel well and satisfied after being active in daily life (cf. [13]). However, the more the preceding bout of physical activities of daily living was autonomously regulated, the more the participants felt better and energized, which has also been shown in a study on students [25]. Thus, the positive association between physical activity in daily living and both valence and energetic arousal is moderated in situ by the degree to which adults aged 50+ regulate their physical activity autonomously.

Furthermore, feelings of competence significantly moderated the association between physical activities of daily living and energetic arousal. Surprisingly, however, this interaction was negative. The more the participants experienced feelings of competence during the preceding bout of physical activities of daily living, the more they felt tired and without energy. This finding might be explained by the effect of different intensities of physical activity. The data showed that the average amount of physical activities of daily living in situations with lower ratings for competence was approximately $30 \%$ lower than that in situations with higher ratings for competence. Thus, greater feelings of competence may be associated with a higher amount of physical activities of daily living and hence with a greater degree of tiredness. In line with this explanation, Oweis and Spinks [44] found that highintensity physical activity resulted in significantly lower levels of energetic arousal. Future studies should confirm the moderating effect of competence for the association between the amount of physical activities of daily living and the affect dimension of energetic arousal.

Relatedness significantly moderated the association between physical activities of daily living and calmness. Specifically, the participants felt more relaxed and calm if they were physically active together with other people. Therefore, being active together with other people weakened the agitating effect of physical activity, indicating that the presence of another person has a calming effect. A possible explanation for this finding is that satisfaction of the need to belong leads to positive feelings of calmness [45], which may counteract the agitating effect of physical activities of daily living.

As a methodological innovation, this study used an ambulatory assessment with activity-triggered e-diaries. As previously demonstrated [27], assessing momentary affective states as a function of the amount of physical activity in daily living situations allows for a greater number of assessments during physically active episodes than using fixed or random sample designs. Specifically, the use of activity-triggered e-diaries maximizes the variation in affective states based on activities of daily living and therefore increases the ability to detect associations between affective states and physical activities of daily living [27].

Notwithstanding this methodological strength, our study nevertheless has a number of limitations. First, although we used a randomization process, we did not achieve a normal distribution for the educational attainment of people aged 50+ in the study that was comparable to that for the general population of these people in Germany [46]. People with high educational attainment are often healthier [47], more satisfied with life [48], and more physically active [5] than other people. Second, we assessed the psychological need for relatedness with the dichotomous measure "being together versus being alone." This measure did not reflect the quality of interpersonal connections and therefore might not be an adequate assessment of the psychological need for belonging. Nevertheless, the data showed that in $80 \%$ of the situations characterized by "being together," the participants rated the other person as very familiar, indicating that being together is associated with feelings of relatedness. Third, although we consider the time dependency of the independent (physical activities of daily living) and the dependent (affective states) variables, the design did not allow for strong causal interpretations, because it is not possible to control for potential confounders with an ambulatory assessment design. Fourth, given 
the fact that the current study assessed physical activities of daily living and that these activities are primarily of low intensities, the relationship between affective states and intensive physical activities as well as the moderating effects of need satisfaction on this association needs to be interpreted with caution and evaluated in future studies.

To the best of our knowledge, no previous study has investigated the moderating effect of all three psychological needs on the within-subject relation between physical activities of daily living and momentary affective states during daily living situations among adults aged 50+. Considering the significant moderating effects, need satisfaction and, especially, autonomy seem to be relevant conditions for enhancing positive affective reactions owing to physical activities. Moreover, being physically active in daily living may be a necessary but not a sufficient condition for strengthening positive affective states. These findings are especially useful for interventions aimed at improving the mental health of adults aged 50+. Further, the positive association between physical activity and affective states will be stronger when activity is more autonomously regulated. To enhance autonomy, people should have the opportunity to choose physical activities that they like and that are fully integrated into their own value system. For instance, a laboratory study showed that self-selected intensities of a treadmill intervention were associated with more positive feelings than prescribed intensities [49]. Thus, an activity intervention that contains detailed and precise recommendations regarding how to do a specific activity should also allow people to decide between different options. The more participants are able to choose between different forms of activity and different kinds of performance, for instance, the more the activity will likely be autonomously regulated and the greater the psychological effect for mental health will be.

Acknowledgments We thank Ulrich Ebner-Primer (Karlsruhe Institute of Technology, Germany) for his substantial contribution to conception and design of the ambulatory assessment study with activity-triggered ediaries.

\section{Compliance with Ethical Standards}

Funding The study was funded by the Deutsche Forschungsgemeinschaft (KA 3279/1-1)

\section{References}

1. Diener E. Subjective well-being: The science of happiness and a proposal for a national index. Am Psychol. 2000; 55: 34-43.

2. Ekkekakis P, Petruzello SJ. Analysis of the affect measurement conundrum in exercise psychology I. Fundamental issues. Psychol Sport Exerc. 2000; 1: 71-88.

3. Lee I-M, Shiroma EJ, Lobelo F, et al. Effects of physical inactivity on major non-communicable diseases worldwide: An analysis of burden of disease and life expectancy. Lancet. 2012; 380: 219-229.
4. Sun F, Norman IJ, While AE. Physical activity in older people: A systematic review. BMC Public Health. 2013; 13: 1-28.

5. Trost SG, Owen N, Bauman AE, Sallis JF, Brown W. Correlates of adults' participation in physical activity: Review and update. Med Sci Sports Exerc. 2002; 34: 1996-2001.

6. Arent SM, Landers DM, Etnier JL. The effects of exercise in mood in older adults: A meta-analytic review. J Aging Phys Act. 2000; 8: 407-430.

7. Netz Y, Wu MJ, Becker BJ, Tenenbaum G. Physical activity and psychological well-being in advanced age: A meta-analysis of intervention studies. Psychol Aging. 2005; 20: 272-284.

8. Vagetti GC, Barbosa FVC, Moreira NB, et al. Association between physical activity and quality of life in the elderly: A systematic review, 2000-2012. Rev Bras Psiquiatr. 2014; 36: 76-88.

9. Dunton GF, Atienza AA, Castro CM, King AC. Using ecological momentary assessment to examine antecedents and correlates of physical activity bouts in adults age $50+$ years: A pilot study. Ann Behav Med. 2009; 38: 249-255.

10. Kanning M, Schlicht W. Be active and become happy: An ecological momentary assessment of physical activity and mood. J Sport Exerc Psychol. 2010; 32: 253-261.

11. Schwerdtfeger A, Eberhardt R, Chmitorz A. Gibt es einen Zusammenhang zwischen Bewegungsaktivität und psychischem Befinden im Alltag? Eine Methodenillustration zum ambulanten Monitoring in der Gesundheitspsychologie. Zeitschrift für Gesundheitspsychologie. 2008; 16: 2-11.

12. Schwerdtfeger A, Eberhardt R, Chmitorz A, Schaller E. Momentary affect predicts bodily movement in daily life: An ambulatory monitoring study. J Sport Exerc Psychol. 2010; 32: 674-693.

13. Kanning M, Ebner-Priemer U, Schlicht W. Using activity triggered e-diaries to reveal the associations between physical activity and affective states in older adult's daily living. Int J Behav Nutr Phys Act. 2015; 12: 1-10.

14. Ortlieb S, Dias A, Gorzelniak L, et al. Exploring patterns of accelerometer-assessed physical activity in elderly people. Int $J$ Behav Nutr Phys Act. 2014; 11: 1-10.

15. Taraldsen K, Chastin SFM, Riphagen II, Vereijken B, Helbostad JL. Physical activity monitoring by use of accelerometer-based bodyworn sensors in older adults: A systematic literature review of current knowledge and applications. Maturitas. 2012; 71: 13-19.

16. Dong L, Block G, Mandel S. Activities contributing to total energy expenditure in the United States: Results from the NHAPS study. Int J Behav Nutr Phys Act. 2004; 1: 4.

17. Ryan RM, Deci EL. Self-determination theory and the facilitation of intrinsic motivation, social development, and well-being. Am Psychol. 2000; 55: 68-78.

18. Deci EL, Ryan RM. The 'what' and 'why' of goal pursuits: Human needs and the self-determination of behavior. Psychol Inq. 2000; 11: 227-268.

19. Ryan RM, Bernstein JH, Brown KW. Weekends, work, and wellbeing: Psychological need satisfactions and day of the week effects on mood, vitality, and physical symptoms. Soc Clin Psychol. 2010; 29: 95-122.

20. Sheldon KM, Elliot AJ. Goal striving, need satisfaction, and longitudinal well-being: The self-concordance model. J Pers Soc Psychol. 1999; 76: 482-497.

21. Reis HT, Sheldon KM, Gable SL, Roscoe J, Ryan RM. Daily wellbeing: The role of autonomy, competence, and relatedness. Pers Soc Psychol Bull. 2000; 26: 419-435.

22. Howell RT, Chenot D, Hill G, Howell CJ. Momentary happiness: The role of psychological need satisfaction. J Happiness Stud. 2011; 12: 1-15.

23. Ebner-Priemer UW, Trull TJ. Ecological momentary assessment of mood disorders and mood dysregulation. Psychol Assess. 2009; 21: 463-475. 
24. Fahrenberg J, Myrtek M, Pawlik K, Perrez M. Ambulatory assessment - monitoring behavior in daily life settings. A behavioralscientific challenge for psychology. Eur J Psychol Assess. 2007; 23: 206-213.

25. Kanning M, Ebner-Priemer UW, Brand R. Autonomous regulation mode moderates the effect of actual physical activity on affective states: An ambulant assessment approach to the role of self-determination. J Sport Exerc Psychol. 2012; 34: 260-269.

26. Hallal PC, Anderson LB, Bull FC, et al. Global physical activity levels: Surveillance progress, pitfalls, and prospects. Lancet. 2012; 380: 247-257.

27. Ebner-Priemer U, Koudela S, Mutz G, Kanning M. Interactive multimodal ambulatory monitoring to investigate the association between physical activity and affect. Front Psychol. 2013; 3: 1-8.

28. Myrtek M. Heart and emotion. Ambulatory monitoring studies in everyday life. Göttingen: Hogrefe; 2004.

29. Russell JA. A circumplex model of affect. J Pers Soc Psychol. 1980; 39: 1161-1178.

30. Thayer RE. The biopsychology of mood and arousal. New York: Oxford University Press; 1989.

31. Schimmack U, Reisenzein R. Experiencing activation: Energetic arousal and tense arousal are not a mixtures of valence and activation. Emotion. 2002; 2: 412-417.

32. Steyer R, Schwenkmezger P, Notz P, Eid M. Der Mehrdimensionale Befindlichekeitsfragebogen (MDBF). Göttingen: Hogrefe; 1997.

33. Wilhelm P, Schoebi D. Assessing mood in daily life. Structural validity, sensitivity to change, and reliability of a short-scale to measure three basic dimensions of mood. Eur J Psychol Assess. 2007; 23: 258-267.

34. Diener E, Emmons RA, Larsen RJ, Griffin S. The satisfaction with life scale. J Person Assess. 1985; 49: 71-75.

35. Pavot W, Diener E. Review of the satisfaction with life scale. Psychol Assess. 1993; 5: 164-172.

36. Gagné $M$. The role of autonomy support and autonomy orientation in prosocial behavior engagement. Motiv Emotion. 2003; 27: 199-223.

37. Raudenbush SW, Bryk A, Congdon R. HLM6: SSI Scientific Software International 2004
38. Nezlek JB. Multilevel modeling in research on personality. In: Robins R, Fraley RC, Krueger R, eds. Handbook of research methods in personality psychology. New York: Guilford; 2007: 502-523.

39. Raudenbush SW, Bryk AS. Hierarchical linear models. 2nd ed. Thousand Oaks: Sage Publications; 2002.

40. Berkemeyer K, Wijndaele K, White T, et al. The descriptive epidemiology of accelerometer-measured physical activity in older adults. Int J Behav Nutr Phys Act. 2016; 7: 1-10.

41. R Core Team. A language and environment for statistical computing. Vienna: R Foundation for Statistical Computing; 2014.

42. Powell R, Allan JL, Johnston DW, et al. Activity and affect: Repeated within-participant assessment in people after joint replacement surgery. Rehabil Psychol. 2009; 54: 83-90.

43. Bossmann T, Kanning M, Koudela S, Hey S, Ebner-Priemer U. The association between short periods of everyday life activities and affective states: A replication using ambulatory assessment. Front Psychol. 2013; 4: 1-7.

44. Oweis P, Spinks W. Biopsychological, affective and cognitive responses to acute physical activity. J Sports Med Phys Fitness. 2001; 41: 528-538.

45. Baumeister RF, Leary MR. The need to belong: Desire for interpersonal attachments as a fundamental human-motivation. Psychol Bull. 1995; 117: 497-529.

46. Dallinger G, Hänsel K, Martin R, Petter M, Habisch R. Datenreport 2013-Ein Sozialbericht für die Bundesrepublik Deutschland. Bonn: Bundeszentrale für politische Bildung; 2013.

47. Kondo N, Sembajwe G, Kawachi I, et al. Income inequality, mortality, and self rated health: A meta analysis of multilevel studies. Br. Med. J. 2009.

48. Diener E, Suh EM, Lucas RE, Smith HL. Subjective well-being: Three decades of progress. Psychol Bull. 1999; 125: 276-302.

49. Rose EA, Parfitt G. A quantitative analysis and qualitative explanation of the individual differences in affective responses to prescribed and self-selected exercise intensities. J Sport Exerc Psychol. 2007; 29: 281-309. 